\title{
STRUCTURE OF BIRD COMMUNITIES FROM CEFA NATURE PARK
}

\author{
Milca PETROVICI * and Attila NAGY ** \\ * West University of Timişoara, Faculty of Chemistry, Biology and Geography, Department of Biology \\ and Chemistry, Pestalozzi Street 16, Timişoara, Romania, RO-300115, milcapetrovici@yahoo.com \\ ** "Grupul Milvus" Bird and Nature Protection Association, Crinului Street 22, Târgu Mureș, Mureș \\ County, Romania, RO-540620, attila.nagy@milvus.ro
}

DOI: 10.1515/trser-2015-0006

KEYWORDS: Romania, Cefa Nature Park, Natura 2000, Birds Directive.

\section{ABSTRACT}

Cefa Nature Park had, even before its establishment, a protection status directed especially towards the bird fauna, in which varied and vast wetlands, forests, meadows and agricultural lands with low agricultural activity are found. The present paper shows the results of a long period of monitoring (1991-2012) the bird fauna from this area. There are currently 78 identified species, protected and recorded in the Annex I from Birds Directive 79/409/CEE. Another 168 species were identified, that are not found on the above list, thus reaching a total number of 246 species (66\% of the total bird fauna of Romania). Quantitative data is also shown with respect to the size of these species population, as well as the belonging to the sedentary or migratory species group. All of the results support the fact that Cefa Nature Park is an area of major importance for bird fauna in the Northwestern territory of Romania, and especially for those species that need wetland habitats for nesting, passage or wintering.

ZUSAMMENFASSUNG: Struktur der Vogelgemeinschaften im Naturpark Cefa.

Der Naturpark Cefa hatte bereits vor seiner Ausweisung als solcher, einen Schutzstatus der insbesondere auf den Vogelbestand dieses Gebietes ausgerichtet war, auf dem sich ausgedehnte, unterschiedliche Feuchtgebietsflächen sowie Wälder, Wiesen und Ackerflächen mit geringer Nutzungsintensität befinden. Die vorliegende Arbeit bringt die Ergebnisse eines langjährigen Monitorings (1991-2012) der Vogelfauna dieses Gebietes. Darunter wurden 78 Arten identifiziert, die einen Schutzstatus haben und im Anhang I der Vogelschutzrichtlinie 79/409/CEE verzeichnet sind. Außerdem wurden weitere 168 Arten identifiziert, die auf obiger Liste nicht enthalten sind, sodass insgesamt 246 Arten festgestellt wurden (66\% der in Rumänien vorkommenden Arten). Hinzu kommen quantitative Angaben bezüglich Populationgröße der Arten sowie deren Zugehörigkeit zur Gruppe der Zug- oder Standvögel. Alle diese Ergebnisse bekräftigen die Tatsache, dass der Naturpark Cefa ein Gebiet von großer Bedeutung für die Vogelfauna im Nordwesten Rumäniens darstellt, insbesondere für die Arten, die zur Brutzeit, während des Durchzugs oder zur Überwinterung Feuchtgebietshabitate benötigen.

REZUMAT: Structura comunităților de păsări din Parcul Natural Cefa.

Parcul Natural Cefa, încă înainte de constituire a avut statut de protecție, care viza în special fauna de păsări din această zonă, în care există întinse și variate suprafețe de zone umede, alături de pădure, pajiști și terenuri arabile pe care se practică o agricultură de intensitate slabă. Lucrarea de față prezintă rezultatele unei monitorizări de lungă durată (19912012) a faunei de păsări din această zonă. Un număr de 78 de specii identificate până în prezent sunt protejate, fiind prezente în Anexa I a Directivei Păsări 79/409/CEE. Pe lângă acestea, un număr de 168 de specii care nu se află pe lista de mai sus, au fost identificate, ajungându-se la un număr total de 246 specii (66\% din totalul faunei de păsări a României). Lucrarea prezintă de asemenea și date cantitative cu privire la mărimea populațiilor acestor specii, precum și apartenența la grupul de specii sedentare sau migratoare. 


\section{INTRODUCTION}

Cefa Nature Park was established in 2010, in the Northwestern part of Romania, on an area of 5002 ha, in order to preserve the natural values of this region. This rich diversity of the habitat and species (Curtean-Bănăduc et al., 2012) is explained by the varied conditions present here. In this area, there are remains of the vast wetlands supplied in the past by the Crişul Repede River and the numerous plain brooks, vast salting meadows, forests, and not lastly, the largest (size-wise) fish-pond complex from the Northwestern Romania.

One of the most important groups for biodiversity preservation is represented by birds; this is why the area was given a protected area status since 1981. That year, the Bihor County Council declared (Resolution 251/20.06.1981) Rădvani Forest a zoological reserve, having the protection of the heron colony as a main goal. Subsequently, the area was given a status of protected natural area of national interest, being declared by Law 5/2000 as the Bird Colony from Rădvani Forest Nature Reserve, with an area of three ha. Beginning in 1989, the ponds from Cefa and Rădvani Forest were listed as Important Bird Areas - AIA - (Munteanu, 2004; Papp and Sándor, 2007; Papp and Fântână, 2008).

Presently, beside the protection status given by establishing the Cefa Nature Park, the area is also part of two Natura 2000 sites: Cefa - code ROSCI0025, (5.413 ha) and Pescăria Cefa - Pădurea Rădvani - code ROSPA0097 (12,253.9 ha).

The goal of this paper is to present the results of a long period monitoring (1991-2012) of the bird fauna from this area. Also, it shows quantitative data with respect to the size of the population of these species, as well as their belonging to the sedentary or migratory species group.

\section{MATERIAL AND METHODS}

Study area. In Cefa Nature Park, on an area of 5002 ha, the following habitats can be found: aquatic habitats (903 ha, 18\% of the total park area), meadows (1,825 ha, 36\%), forest (238 ha, 5\%) and arable land (937 ha, 19\%).

Aquatic habitats. The establishment of Cefa fishponds was favored by its location on lowland, subject to flooding which led to marsh forming. After building the Collector Canal (in 1899), the fishponds were created and administrated today by private business. On the fishery area there are 47 basins, with a depth of 1-1.5 $\mathrm{m}$ and a total water surface of approximative 663 ha. Besides the fishponds, there can also be found marshes, natural pools, and wet fields networked by canals and ditches.

Meadow habitats. They are used as pastures (1,624 ha, 32\% of the total park area) and hayfields (201 ha, 4\%). After canalling and draining, they were salinated thus transforming into saltings, scattered all over the park, and covered with halofile secondary vegetation. These saltings have striped characteristics, or a concentric display of the vegetation, according to the salt concentration and the hydric regime of the soil.

Forest habitat. Rădvani Forest is the remnants of the old plain oak forest covering the Crişurilor Plain, alternating with steppe meadows. The average age of the main forest body (Cefa - Ateaş) is between 65-70 years old, with the oldest trees - English oak Quercus robur at 80 years old. Cefa's forest body has an average age of 65 years old, with its oldest trees at 70 years old.

Arable land. Even though the Cefa Nature Park territory is an anthropized habitat, the agricultural surfaces support a rich biodiversity, not being exploited in an intensive system. Many of these surfaces show a high degree of abandonment (because of their high wetness and salting). 
The paper was elaborated based on observations made in the Cefa Nature Park area starting in 1991. During this period, there have been numerous bird monitoring actions in the area. For the inventory of bird species, direct observations, and methods of line and point transects have been used (Sutherland et al., 2004). Observation started at 6:30-7:00 a.m. and continued until evening at sunset, around 8:00 p.m.

For inventory and monitoring activities, binoculars (Minox HG 10x52 and Leica Ultravid 10x42 HD), telescopes (Zeiss DiaScope 85, 20-60x85) and bird field guides were used (Bruun et al., 1999; Svensson et al., 2010). All observations were noted in the field. Later, centralization and systematization of the data were performed.

\section{RESULTS AND DISCUSSION}

Bird species identified in the Cefa Nature Park are presented within table 1, highlighting the breeding pairs, number of individuals from resident species, as well as wintering and staging species. Binary taxonomy was updated by Avisabe - The world bird database $(*)$.

Table 1: Birds species from Cefa Nature Park, 1991-2012. A: abundant (species existing in over $75 \%$ of systematic observations - s.o.), C: common (species existing in over $51-75 \%$ of s.o.), RC: relatively common (species existing in over $26-50 \%$ of s.o.), R: rare (species existing in over 11-25\% of s.o.), VR: very rare (species existing in over 6-10\% of s.o.), S: sporadically (species existing in over 1-5\% of s.o.), ER: erratic (species existing only exceptionally, under $1 \%$ of s.o.).

\begin{tabular}{|c|c|c|c|c|c|}
\hline \multirow{3}{*}{$\begin{array}{l}\text { Natura } \\
2000 \\
\text { Code }\end{array}$} & \multirow{3}{*}{ Species } & \multicolumn{4}{|c|}{ Population size } \\
\hline & & \multirow{2}{*}{$\begin{array}{l}\text { Resident } \\
\text { species }\end{array}$} & \multicolumn{3}{|c|}{ Migratory species } \\
\hline & & & $\begin{array}{c}\text { Breeding } \\
\text { (pairs) }\end{array}$ & $\begin{array}{c}\text { Wintering } \\
\text { (individuals) }\end{array}$ & $\begin{array}{c}\text { Staging } \\
\text { (individuals) }\end{array}$ \\
\hline \multicolumn{6}{|c|}{ Species from Annex I - Birds Directive 79/409/CEE } \\
\hline A001 & 1. Gavia stellata & & & & $1-3$ \\
\hline A002 & 2. Gavia arctica & & & & $2-10$ \\
\hline A393 & 3. Phalacrocorax pygmaeus & & & & $4-25$ \\
\hline A020 & 4. Pelecanus crispus & & & & $0-1$ \\
\hline A021 & 5. Botaurus stellaris & & $6-10$ & $0-2$ & $10-15$ \\
\hline A022 & 6. Ixobrychus minutus & & $40-50$ & & $200-400$ \\
\hline A023 & 7. Nycticorax nycticorax & & $120-150$ & $0-1$ & $600-900$ \\
\hline A024 & 8. Ardeola ralloides & & $3-7$ & & $15-40$ \\
\hline A026 & 9. $\quad$ Egretta garzetta & & $5-14$ & & $250-400$ \\
\hline A027 & 10. Egretta alba & & $8-15$ & $2-6$ & $400-700$ \\
\hline A029 & 11. Ardea purpurea & & $8-10$ & & $80-120$ \\
\hline A030 & 12. Ciconia nigra & & $0-1$ & & $12-20$ \\
\hline A031 & 13. Ciconia ciconia & & $2-4$ & & \\
\hline A032 & 14. Plegadis falcinellus & & & & $0-5$ \\
\hline A034 & 15. Platalea leucorodia & & & & $150-360$ \\
\hline A038 & 16. Cygnus cygnus & & & & $0-4$ \\
\hline A042 & 17. Anser erythropus & & & & $0-3$ \\
\hline A396 & 18. Branta ruficollis & & & & $0-9$ \\
\hline A397 & 19. Tadorna ferruginea & & & & $0-4$ \\
\hline
\end{tabular}


Table 1 (continuing): Birds species from Cefa Nature Park, 1991-2012.

\begin{tabular}{|c|c|c|c|c|c|}
\hline \multirow{3}{*}{$\begin{array}{l}\text { Natura } \\
2000 \\
\text { Code }\end{array}$} & \multirow{3}{*}{ Species } & \multicolumn{4}{|c|}{ Population size } \\
\hline & & \multirow{2}{*}{$\begin{array}{c}\text { Resident } \\
\text { species }\end{array}$} & \multicolumn{3}{|c|}{ Migratory species } \\
\hline & & & $\begin{array}{c}\text { Breeding } \\
\text { (pairs) }\end{array}$ & $\begin{array}{c}\text { Wintering } \\
\text { (individuals) }\end{array}$ & $\begin{array}{c}\text { Staging } \\
\text { (individuals) }\end{array}$ \\
\hline A060 & 20. Aythya nyroca & & $15-35$ & & $150-380$ \\
\hline A068 & 21. Mergus albellus & & & & $25-80$ \\
\hline A072 & 22. Pernis apivorus & & $0-1$ & & $15-20$ \\
\hline A074 & 23. Milvus migrans & & $0-1$ & & $5-8$ \\
\hline A075 & 24. Milvus milvus & & & & $0-1$ \\
\hline A076 & 25. Haliaeetus albicilla & & & $15-55$ & \\
\hline A080 & 26. Circaetus gallicus & & & & $0-2$ \\
\hline A081 & 27. Circus aeruginosus & & $8-12$ & $0-2$ & $150-220$ \\
\hline A082 & 28. Circus cyaneus & & & $2-4$ & $10-100$ \\
\hline A083 & 29. Circus macrourus & & & & $0-2$ \\
\hline A084 & 30. Circus pygargus & & $0-2$ & & $12-18$ \\
\hline A403 & 31. Buteo rufinus & & & & $1-3$ \\
\hline A089 & 32. Aquila pomarina & & $0-1$ & & $0-2$ \\
\hline A090 & 33. Aquila clanga & & & & 1 \\
\hline A404 & 34. Aquila heliaca & & & & $0-3$ \\
\hline A092 & 35. Hieraaetus pennatus & & & & $0-1$ \\
\hline A094 & 36. Pandion haliaetus & & & & $5-12$ \\
\hline A097 & 37. Falco vespertinus & & & & $0-12$ \\
\hline A098 & 38. Falco columbarius & & & $1-3$ & $10-18$ \\
\hline A511 & 39. Falco cherrug & & $0-1$ & & $4-8$ \\
\hline A103 & 40. Falco peregrinus & & & & $3-5$ \\
\hline A119 & 41. Porzana porzana & & $\mathrm{R}$ & & \\
\hline A120 & 42. Porzana parva & & $\mathrm{R}$ & & \\
\hline A122 & 43. Crex crex & & $0-4$ & & \\
\hline A127 & 44. Grus grus & & & & $0-90$ \\
\hline A129 & 45. Otis tarda & & & & $0-2$ \\
\hline A131 & 46. Himantopus himantopus & & $0-3$ & & $2-30$ \\
\hline A132 & 47. Recurvirostra avosetta & & $0-12$ & & $60-120$ \\
\hline A140 & 48. Pluvialis apricaria & & & & $250-800$ \\
\hline A149 & 49. Calidris alpina & & & & $600-1,000$ \\
\hline A151 & 50. Philomachus pugnax & & & & $2,200-7,000$ \\
\hline A154 & 51. Gallinago media & & & & VR \\
\hline A157 & 52. Limosa lapponica & & & & $0-2$ \\
\hline A166 & 53. Tringa glareola & & & & $800-1,400$ \\
\hline A170 & 54. Phalaropus lobatus & & & & $0-1$ \\
\hline A176 & 55. Ichthyaetus melanocephalus & & & & $0-1$ \\
\hline A177 & 56. Hydrocoloeus (Larus) minutus & & & & $2-30$ \\
\hline A189 & 57. Gelochelidon nilotica & & & & $0-1$ \\
\hline A190 & 58. Hydroprogne (Sterna) caspia & & & & $0-1$ \\
\hline A193 & 59. Sterna hirundo & & $0-20$ & & $80-240$ \\
\hline A195 & 60. Sterna albifrons & & & & $0-1$ \\
\hline
\end{tabular}


Table 1 (continuing): Birds species from Cefa Nature Park, 1991-2012.

\begin{tabular}{|c|c|c|c|c|c|}
\hline \multirow{3}{*}{$\begin{array}{l}\text { Natura } \\
2000 \\
\text { Code }\end{array}$} & \multirow{3}{*}{ Species } & \multicolumn{4}{|c|}{ Population size } \\
\hline & & \multirow{2}{*}{$\begin{array}{l}\text { Resident } \\
\text { species }\end{array}$} & \multicolumn{3}{|c|}{ Migratory species } \\
\hline & & & $\begin{array}{l}\text { Breeding } \\
\text { (pairs) }\end{array}$ & $\begin{array}{c}\text { Wintering } \\
\text { (individuals) }\end{array}$ & $\begin{array}{c}\text { Staging } \\
\text { (individuals) }\end{array}$ \\
\hline A196 & 61. Chlidonias hybridus & & $5-240$ & & $350-650$ \\
\hline A197 & 62. Chlidonias niger & & $0-10$ & & $45-300$ \\
\hline A222 & 63. Asio flammeus & & $0-1$ & & $0-20$ \\
\hline A229 & 64. Alcedo atthis & & $0-2$ & $2-4$ & \\
\hline A231 & 65. Coracias garrulus & & $0-1$ & & $0-8$ \\
\hline A234 & 66. Picus canus & $0-1$ & & & \\
\hline A236 & 67. Dryocopus martius & $1-2$ & & & \\
\hline A429 & 68. Dendrocopos syriacus & $1-4$ & & & \\
\hline A238 & 69. Dendrocopos medius & $2-4$ & & & \\
\hline A245 & 70. Galerida cristata & $\mathrm{C}$ & & & \\
\hline A246 & 71. Lullula arborea & & $1-2$ & & \\
\hline A255 & 72. Anthus campestris & & $0-2$ & & \\
\hline A272 & 73. Luscinia svecica & & $2-4$ & & \\
\hline A293 & 74. Acrocephalus melanopogon & & $1-3$ & & \\
\hline A307 & 75. Sylvia nisoria & & $1-4$ & & \\
\hline A321 & 76. Ficedula albicollis & & $1-2$ & & \\
\hline A338 & 77. Lanius collurio & & C & & \\
\hline A339 & 78. Lanius minor & & $6-12$ & & \\
\hline \multicolumn{6}{|c|}{ Species which are not in Annex I from Birds Directive 79/409/CEE } \\
\hline A004 & 1. Tachybaptus ruficollis & & $12-30$ & & $600-1,000$ \\
\hline A005 & 2. Podiceps cristatus & & $120-200$ & & $600-800$ \\
\hline A006 & 3. Podiceps grisegena & & $1-2$ & & $15-20$ \\
\hline A008 & 4. Podiceps nigricollis & & $3-18$ & & $120-200$ \\
\hline A017 & 5. Phalacrocorax carbo & & & & $1,200-2,500$ \\
\hline A025 & 6. Bubulcus ibis & & & & ER \\
\hline A028 & 7. Ardea cinerea & & $293-370$ & & $800-1,200$ \\
\hline A036 & 8. Cygnus olor & & $0-1$ & & $4-24$ \\
\hline A039 & 9. Anser fabalis & & & & ER \\
\hline A041 & 10. Anser albifrons & & & & $5,000-15,000$ \\
\hline A043 & 11. Anser anser & & $10-25$ & & $250-1,200$ \\
\hline A048 & 12. Tadorna tadorna & & & & $10-20$ \\
\hline A050 & 13. Anas penelope & & & & $600-1,000$ \\
\hline A051 & 14. Anas strepera & & $10-12$ & & $120-360$ \\
\hline A052 & 15. Anas crecca & & & & $6,000-10,000$ \\
\hline A053 & 16. Anas platyrhynchos & & $20-30$ & $20-50$ & $4,000-7,000$ \\
\hline A054 & 17. Anas acuta & & & & $140-260$ \\
\hline A055 & 18. Anas querquedula & & $4-7$ & & $800-2,000$ \\
\hline A056 & 19. Anas clypeata & & & & $1,000-2,000$ \\
\hline A058 & 20. Netta rufina & & $0-2$ & & $0-8$ \\
\hline A059 & 21. Aythya ferina & & $75-120$ & $8-16$ & $3,000-8,000$ \\
\hline
\end{tabular}


Table 1 (continuing): Birds species from Cefa Nature Park, 1991-2012.

\begin{tabular}{|c|c|c|c|c|c|}
\hline \multirow{3}{*}{$\begin{array}{l}\text { Natura } \\
2000 \\
\text { Code }\end{array}$} & \multirow{3}{*}{ Species } & \multicolumn{4}{|c|}{ Population size } \\
\hline & & \multirow{2}{*}{$\begin{array}{c}\text { Resident } \\
\text { species }\end{array}$} & \multicolumn{3}{|c|}{ Migratory species } \\
\hline & & & $\begin{array}{c}\text { Breeding } \\
\text { (pairs) }\end{array}$ & $\begin{array}{c}\text { Wintering } \\
\text { (individuals) }\end{array}$ & $\begin{array}{c}\text { Staging } \\
\text { (individuals) }\end{array}$ \\
\hline A061 & 22. Aythya fuligula & & $0-1$ & & $400-800$ \\
\hline A067 & 23. Bucephala clangula & & & & $120-250$ \\
\hline A069 & 24. Mergus serrator & & & & $0-8$ \\
\hline A070 & 25. Mergus merganser & & & & $10-28$ \\
\hline A086 & 26. Accipiter nisus & & $0-1$ & $4-10$ & \\
\hline A085 & 27. Accipiter gentilis & & $2-3$ & & \\
\hline A087 & 28. Buteo buteo & & 3-5 & $12-30$ & \\
\hline A088 & 29. Buteo lagopus & & & $3-6$ & \\
\hline A096 & 30. Falco tinnunculus & & $4-10$ & $8-18$ & \\
\hline A099 & 31. Falco subbuteo & & $1-2$ & & \\
\hline A113 & 32. Coturnix coturnix & & $10-25$ & & \\
\hline A115 & 33. Phasianus colchicus & & $\mathrm{C}$ & & \\
\hline A118 & 34. Rallus aquaticus & & $10-30$ & & \\
\hline A123 & 35. Gallinula chloropus & & $160-240$ & & $250-320$ \\
\hline A125 & 36. Fulica atra & & $120-200$ & & $8,000-14,000$ \\
\hline A130 & 37. Haematopus ostralegus & & & & $0-2$ \\
\hline A136 & 38. Charadrius dubius & & $5-12$ & & $60-140$ \\
\hline A137 & 39. Charadrius hiaticula & & & & $4-18$ \\
\hline A141 & 40. Pluvialis squatarola & & & & $60-120$ \\
\hline A142 & 41. Vanellus vanellus & & $25-60$ & & $6,000-9,000$ \\
\hline A144 & 42. Calidris alba & & & & $6-20$ \\
\hline A149 & 43. Calidris alpina & & & & $\mathrm{S}$ \\
\hline $\begin{array}{c}\text { Without } \\
\text { code }\end{array}$ & 44. Calidris melanotos & & & & VR \\
\hline A145 & 45. Calidris minuta & & & & $360-500$ \\
\hline A146 & 46. Calidris temminckii & & & & $20-60$ \\
\hline A147 & 47. Calidris ferruginea & & & & $160-220$ \\
\hline A150 & 48. Limicola falcinellus & & & & $2-6$ \\
\hline A152 & 49. Lymnocryptes minimus & & & & $30-60$ \\
\hline A153 & 50. Gallinago gallinago & & $0-6$ & & $300-450$ \\
\hline A155 & 51. Scolopax rusticola & & $0-1$ & & $8-16$ \\
\hline A156 & 52. Limosa limosa & & & & $6,000-9,000$ \\
\hline A158 & 53. Numenius phaeopus & & & & $800-1,200$ \\
\hline A160 & 54. Numenius arquata & & $0-7$ & & $1,400-1,800$ \\
\hline A161 & 55. Tringa erythropus & & & & $1,500-2,500$ \\
\hline A162 & 56. Tringa totanus & & $1-7$ & & $120-280$ \\
\hline A163 & 57. Tringa stagnatilis & & & & $40-60$ \\
\hline A164 & 58. Tringa nebularia & & & & $240-460$ \\
\hline A165 & 59. Tringa ochropus & & & & $140-300$ \\
\hline A168 & 60. Actitis hypoleucos & & & $0-1$ & $150-230$ \\
\hline A169 & 61. Arenaria interpres & & & & $0-1$ \\
\hline A171 & 62. Phalaropus fulicarius & & & & $0-8$ \\
\hline
\end{tabular}


Table 1 (continuing): Birds species from Cefa Nature Park, 1991-2012.

\begin{tabular}{|c|c|c|c|c|c|}
\hline \multirow{3}{*}{$\begin{array}{l}\text { Natura } \\
2000 \\
\text { Code }\end{array}$} & \multirow{3}{*}{ Species } & \multicolumn{4}{|c|}{ Population size } \\
\hline & & \multirow{2}{*}{$\begin{array}{c}\text { Resident } \\
\text { species }\end{array}$} & \multicolumn{3}{|c|}{ Migratory species } \\
\hline & & & $\begin{array}{c}\text { Breeding } \\
\text { (pairs) }\end{array}$ & $\begin{array}{c}\text { Wintering } \\
\text { (individuals) }\end{array}$ & $\begin{array}{c}\text { Staging } \\
\text { (individuals) }\end{array}$ \\
\hline A179 & 63. Chroicocephalus ridibundus & & $0-30$ & & $15,000-35,000$ \\
\hline A182 & 64. Larus canus & & & & $360-700$ \\
\hline A183 & 65. Larus fuscus & & & & $4-12$ \\
\hline A184 & 66. Larus argentatus & & & & VR \\
\hline A604 & 67. Larus michahellis & & & & VR \\
\hline A184 & 68. Larus cachinnans & & & & $1,000-2,000$ \\
\hline A198 & 69. Chlidonias leucopterus & & & & $45-60$ \\
\hline A207 & 70. Columba oenas & & $1-3$ & & VR \\
\hline A206 & 71. Columba livia & & $\mathrm{C}$ & & \\
\hline A208 & 72. Columba palumbus & & $4-10$ & & $\mathrm{R}$ \\
\hline A210 & 73. Streptopelia turtur & & $3-9$ & & VR \\
\hline A209 & 74. Streptopelia decaocto & & & & \\
\hline A212 & 75. Cuculus canorus & & $\mathrm{RC}$ & & $\mathrm{RC}$ \\
\hline A214 & 76. Otus scops & & $1-3$ & & \\
\hline A221 & 77. Asio otus & & $2-3$ & & \\
\hline A218 & 78. Athene noctua & & $2-3$ & & \\
\hline A213 & 79. Tyto alba & & 3-4 & & \\
\hline A226 & 80. Apus apus & & & & $400-800$ \\
\hline A230 & 81. Merops apiaster & & & & $200-600$ \\
\hline A235 & 82. Picus viridis & & $\mathrm{C}$ & & \\
\hline A237 & 83. Dendrocopos major & & $\mathrm{C}$ & & \\
\hline A240 & 84. Dendrocopos minor & & $\mathrm{C}$ & & \\
\hline A232 & 85. Upupa epops & & $1-2$ & & $25-50$ \\
\hline A233 & 86. Jynx torquilla & & $1-3$ & & $15-25$ \\
\hline A247 & 87. Alauda arvensis & & $\mathrm{C}$ & & $\mathrm{RC}$ \\
\hline A249 & 88. Riparia riparia & & & & $3,000-8,000$ \\
\hline A251 & 89. Hirundo rustica & & $\mathrm{C}$ & & \\
\hline A253 & 90. Delichon urbicum (urbica) & & $\mathrm{C}$ & & $8,000-14,000$ \\
\hline A256 & 91. Anthus trivialis & & $1-4$ & & \\
\hline A257 & 92. Anthus pratensis & & & & $20-50$ \\
\hline A258 & 93. Anthus cervinus & & & & $2-10$ \\
\hline A259 & 94. Anthus spinoletta & & & $1-5$ & $400-800$ \\
\hline A260 & 95. Motacilla flava flava & & $70-180$ & & $1,000-2,000$ \\
\hline A261 & 96. Motacilla cinerea & & & & $\mathrm{S}$ \\
\hline A262 & 97. Motacilla alba & & $20-55$ & & $1,500-3,000$ \\
\hline A263 & 98. Bombycilla garrulus & & & & ER \\
\hline A265 & 99. Troglodytes troglodytes & & $\mathrm{C}$ & & \\
\hline A266 & 100. Prunella modularis & & $\mathrm{C}$ & & \\
\hline A269 & 101. Erithacus rubecula & & A & & $\mathrm{RC}$ \\
\hline A270 & 102. Luscinia luscinia & & ER & & ER \\
\hline
\end{tabular}


Table 1 (continuing): Birds species from Cefa Nature Park, 1991-2012.

\begin{tabular}{|c|c|c|c|c|c|}
\hline \multirow{3}{*}{$\begin{array}{l}\text { Natura } \\
2000 \\
\text { Code }\end{array}$} & \multirow{3}{*}{ Species } & \multicolumn{4}{|c|}{ Population size } \\
\hline & & \multirow{2}{*}{$\begin{array}{c}\text { Resident } \\
\text { species }\end{array}$} & \multicolumn{3}{|c|}{ Migratory species } \\
\hline & & & $\begin{array}{c}\text { Breeding } \\
\text { (pairs) }\end{array}$ & $\begin{array}{c}\text { Wintering } \\
\text { (individuals) }\end{array}$ & $\begin{array}{c}\text { Staging } \\
\text { (individuals) }\end{array}$ \\
\hline A271 & 103. Luscinia megarhynchos & & $\mathrm{RC}$ & & $\mathrm{RC}$ \\
\hline A273 & 104. Phoenicurus ochruros & & $\mathrm{C}$ & & $\mathrm{RC}$ \\
\hline A274 & 105. Phoenicurus phoenicurus & & & & VR \\
\hline A275 & 106. Saxicola rubetra & & VR & & VR \\
\hline A276 & 107. Saxicola torquata & & $\mathrm{R}$ & & $\mathrm{R}$ \\
\hline A278 & 108. Oenanthe oenanthe & & $\mathrm{R}$ & & $\mathrm{S}$ \\
\hline A283 & 109. Turdus merula & A & & & \\
\hline A284 & 110. Turdus pilaris & & & & $\mathrm{R}$ \\
\hline A285 & 111. Turdus philomelos & & $\mathrm{R}$ & & $\mathrm{RC}$ \\
\hline A286 & 112. Turdus iliacus & & & & ER \\
\hline A287 & 113. Turdus viscivorus & & VR & & $\mathrm{R}$ \\
\hline A290 & 114. Locustella naevia & & $0-2$ & & ER \\
\hline A291 & 115. Locustella fluviatilis & & VR & & VR \\
\hline A292 & 116. Locustella luscinioides & & RC & & $\mathrm{RC}$ \\
\hline A295 & 117. Acrocephalus shoenobaenus & & A & & A \\
\hline A296 & 118. Acrocephalus palustris & & $\mathrm{RC}$ & & $\mathrm{RC}$ \\
\hline A297 & 119. Acrocephalus scirpaceus & & A & & A \\
\hline A298 & 120. Acrocephalus arundinaceus & & A & & A \\
\hline A438 & 121. Hippolais pallida & & & & ER \\
\hline A299 & 122. Hippolais icterina & & VR & & $\mathrm{RC}$ \\
\hline A308 & 123. Sylvia curruca & & $\mathrm{RC}$ & & $\mathrm{RC}$ \\
\hline A309 & 124. Sylvia communis & & $\mathrm{RC}$ & & $\mathrm{RC}$ \\
\hline A310 & 125. Sylvia borin & & $\mathrm{R}$ & & $\mathrm{R}$ \\
\hline A311 & 126. Sylvia atricapilla & & $\mathrm{RC}$ & & $\mathrm{C}$ \\
\hline A314 & 127. Phylloscopus sibilatrix & & VR & & $\mathrm{R}$ \\
\hline A315 & 128. Phylloscopus collybita & & RC & & $\mathrm{C}$ \\
\hline A316 & 129. Phylloscopus trochilus & & $\mathrm{S}$ & & $\mathrm{C}$ \\
\hline A317 & 130. Regulus regulus & & & & $\mathrm{S}$ \\
\hline A318 & 131. Regulus ignicapillus & & & & $\mathrm{S}$ \\
\hline A319 & 132. Muscicapa striata & & $\mathrm{R}$ & & $\mathrm{RC}$ \\
\hline A324 & 133. Aegithalos caudatus & & $\mathrm{C}$ & & \\
\hline A330 & 134. Parus major & & $\mathrm{C}$ & & \\
\hline A329 & 135. Cyanistes (Parus) caeruleus & & $\mathrm{C}$ & & \\
\hline A325 & 136. Poecile (Parus) palustris & & $\mathrm{C}$ & & \\
\hline A323 & 137. Panurus biarmicus & & $\mathrm{C}$ & & \\
\hline A336 & 138. Remiz pendulinus & & $20-40$ & & $\mathrm{RC}$ \\
\hline A332 & 139. Sitta europaea & & $\mathrm{C}$ & & \\
\hline A337 & 140. Oriolus oriolus & & $6-12$ & & VR \\
\hline A340 & 141. Lanius excubitor & & & $15-45$ & \\
\hline A342 & 142. Garrulus glandarius & & A & & \\
\hline A343 & 143. Рica pica & & A & & \\
\hline
\end{tabular}


Table 1 (continuing): Birds species from Cefa Nature Park, 1991-2012.

\begin{tabular}{|c|c|c|c|c|c|}
\hline \multirow{3}{*}{$\begin{array}{l}\text { Natura } \\
2000 \\
\text { Code }\end{array}$} & \multirow{3}{*}{ Species } & \multicolumn{4}{|c|}{ Population size } \\
\hline & & \multirow{2}{*}{$\begin{array}{l}\text { Resident } \\
\text { species }\end{array}$} & \multicolumn{3}{|c|}{ Migratory species } \\
\hline & & & $\begin{array}{c}\text { Breeding } \\
\text { (pairs) }\end{array}$ & $\begin{array}{c}\text { Wintering } \\
\text { (individuals) }\end{array}$ & $\begin{array}{c}\text { Staging } \\
\text { (individuals) }\end{array}$ \\
\hline A347 & 144. Corvus monedula & & A & & \\
\hline A348 & 145. Corvus frugilegus & & A & & \\
\hline A349 & 146. Corvus cornix & & A & & \\
\hline A350 & 147. Corvus corax & & $5-8$ & & \\
\hline A351 & 148. Sturnus vulgaris & & $\mathrm{C}$ & & A \\
\hline A353 & 149. Sturnus roseus & & & & ER \\
\hline A354 & 150. Passer montanus & & A & & \\
\hline A356 & 151. Passer domesticus & & A & & \\
\hline A359 & 152. Fringilla coelebs & $\mathrm{C}$ & & & \\
\hline A360 & 153. Fringilla montifringilla & & & $\mathrm{RC}$ & \\
\hline A361 & 154. Serinus serinus & & $\mathrm{R}$ & & $\mathrm{R}$ \\
\hline A363 & 155. Carduelis chloris & $\mathrm{RC}$ & & & \\
\hline A364 & 156. Carduelis carduelis & $\mathrm{RC}$ & & & \\
\hline A365 & 157. Carduelis spinus & & & ER & \\
\hline A366 & 158. Carduelis cannabina & $\mathrm{S}$ & & & \\
\hline A367 & 159. Carduelis flavirostris & & & ER & \\
\hline A368 & 160. Carduelis flammea & & & & ER \\
\hline A369 & 161. Loxia curvirostris & & & & ER \\
\hline A372 & 162. Pyrrhula pyrrhula & & & & VR \\
\hline A373 & 163. Coccothraustes coccothraustes & $\mathrm{R}$ & & & \\
\hline A374 & 164. Calcarius lapponicus & & & ER & \\
\hline A375 & 165. Plectrophenax nivalis & & & & ER \\
\hline A376 & 166. Emberiza citrinella & & $\mathrm{C}$ & & \\
\hline A381 & 167. Emberiza schoeniclus & & $\mathrm{C}$ & & \\
\hline A383 & 168. Miliaria calandra & & $\mathrm{C}$ & & \\
\hline
\end{tabular}

\section{Migration birds in the spring/fall passages from Cefa Nature Park}

Cefa fishponds, as well as those from the surrounding areas, provide (thanks to their placement on the Pannonic-Bulgarian migration path) ideal habitats for feeding, weight gaining and rest for thousands of birds during spring and fall migrations.

A characteristic of the spring passage is that it starts very early due to mild winters and short time frosts. Cefa Nature Park conditions provide for birds in the spring passage with resting habitats. First on the water surfaces, on the bottoms of the ponds emptied during this time of the year and on pastures, in the two forest bodies and neighbouring arable lands.

Fall passage starts early at Cefa, August-December, depending on the temperature. Favorable conditions, especially for shorebirds, occur during this time of the year by industrial fishing that requires water removal from ponds, leaving only shallow water layers. Thus, on such ponds, thousands of birds can be recorded, belonging to species such as Eurasian Curlew Numenius arquata and Whimbrel N. phaeopus, Northern Lapwing Vanellus vanellus, Blacktailed Godwit Limosa limosa, Ruff Philomachus pugnax, European Golden Plover Pluvialis apricaria, Gray Plover P. squatarola, Spotted Redshank Tringa erythropus, Common Redshank T. totanus, Marsh Sandpiper T. stagnatilis, Greenshank T. nebularia, Green Sandpiper T. ochropus, Wood Sandpiper T. glareola, Common Sandpiper Actitis hypoleucos, 
Little Ringed Plover Charadrius dubius, Ringed Plover C. hiaticula, Sanderling Calidris alba, Little Stint C. minuta, Temminck's Stint C. temminckii, Curlew Sandpiper C. ferruginea, Dunlin C. alpina, Black-winged Stilt Himantopus himantopus, Avocet Recurvirostra avosetta, Jack Snipe Lymnocryptes minimus, Common Snipe Gallinago gallinago.

Also attracted by the trophic supply of the ponds with decreasing water levels, during their passage, there are species like the gulls and terns: Black-headed Gull Chroicocephalus (Larus) ridibundus (15,000-35,000 individuals), Caspian Gull Larus cachinnans (1,000-2,000 individuals) Common Gull L. canus (360-700 individuals), Lesser Black-backed Gull L. fuscus, Little Gull Hydrocoloeus (Larus) minutus, Common Tern Sterna hirundo (80-240 individuals), Whiskered Tern Chlidonias hybridus (350-650 individuals), White-winged Tern Chlidonias leucopterus (45-60 individuals).

Eurasian Spoonbills Platalea leucorodia (sometimes even Glossy Ibis Plegadis falcinellus), which are nesting in the Kis-Sarret area from Körös-Maros National Park, perform in large groups during migration on ponds drained of water.

A lot less, but regularly recorded during their passage on the emptied ponds, there are individuals of species: Bar-tailed Godwit Limosa lapponica, Little Tern Sterna albifrons, Caspian Tern Hydroprogne (Sterna) caspia, Gull-billed Tern Gelochelidon nilotica, Ruddy Turnstone Arenaria interpres, Red-necked Phalarope Phalaropus lobatus, Red Phalarope Phalaropus fulicarius, Broad-billed Sandpiper Limicola falcinellus, Eurasian Oystercatcher Haematopus ostralegus, Mediterranean Gull Ichthyaetus (Larus) melanocephalus.

Surfaces with reed provide night habitats during fall for about 3,000-8,000 of the Sand Martin Riparia riparia and 8,000-14,000 Northern House Martin Delichon urbicum (urbica).

In the ponds area, even if not directly influenced by them, can be recorded during their fall passage, 200-600 of European Bee-eater Merops apiaster, 400-800 Water Pipit Anthus spinoletta, 1,000-2,000 Yellow Wagtail Motacilla flava, and 1,500-3,000 White Wagtail M. alba.

Fields covered with hay, pastures and agricultural crops are the resting and/or feeding place outside the nesting period for Greater White-fronted Goose Anser albifrons (over 10,000 individuals) and the endangered species: Lesser White-fronted Goose Anser erythropus (3 individuals), Red-breasted Goose Branta ruficollis (up to nine individuals), Common Crane Grus grus (90 individuals), and Short-eared Owl Asio flammeus (20 individuals).

Of those recorded as extremely rare during winter or in passage are: Cattle Egret Bubulcus ibis, Bohemian Waxwing Bombycilla garrulus, Common Crossbill Loxia curvirostra, Snow Bunting Plectrophenax nivalis, Lapland Bunting Calcarius lapponicus and Dalmatian Pelican Pelecanus crispus. Ölvedi Szilárd (personal communication) identified once (October 2011), during a bird watching marathon species Pectoral Sandpiper Calidris melanotos.

Species' richness attracts predatory bird species due to a great number of birds found during their migration passage and in winter. Thus, recorded during passage going through this area were: five to eight individuals of Black Kite Milvus migrans, one Red Kite Milvus milvus, 15-55 White-tailed Eagle Haliaetus albicilla, two Short-toed Eagle Circaetus gallicus, 150220 Western Marsh Harrier Circus aeruginosus, 90-100 Hen Harrier C. cyaneus, two Pallid Harrier C. macrouros, 12-18 Montagu's Harrier C. pygargus, two Lesser Spotted Eagle Aquila pomarina, one Greater Spotted Eagle $A$. clanga, one to three Eastern Imperial Eagle $A$. heliaca, one Booted Eagle Aquila Hieraaetus pennatus, 10-12 Osprey Pandion haliaetus, 12 Red-footed Falcon Falco vespertinus, 10-18 Merlin F. columbarius, four to eight Saker Falcon $F$. cherrug, three to five Peregrine Falcon F. peregrinus, 10-18 Common Kestrel $F$. tinnunculus, four to nine Eurasian Sparrowhawk Accipiter nisus, 12-30 Common Buzzard Buteo buteo, three to six Rough-legged Buzzard B. lagopus, one to three Long-legged Buzzard B. rufinus. 


\section{Breeding birds species in Cefa Nature Park \\ Breeding bird species in Rădvani Forest}

Rădvani Forest is an adequate habitat for nesting to a great number of herons. Also, it displays proper conditions for nesting of several diurnal and nocturnal predatory species, as well as multitudes of Passeriformes.

In Rădvani Forest, there is a mixed colony of herons, already known from the '40s (Munteanu, 2000). There are breeding together: Grey Heron Ardea cinerea (293-370 pairs), Night Heron Nycticorax nycticorax (120-150 pairs) and Little Egret Egretta garzetta (5-14 pairs). The colony is installed in about 100 English Oak Quercus robur and European Ash Fraxinus excelsior trees, with heights ranging from 20-25 m. The number of nests vary from one to eight on a tree with an average of four to five nests per tree.

In the Rădvani forest, there is a pair of Black Stork Ciconia nigra, nesting here, even though not always successfully. The habitat is adequate for this species, necessitating the presence of an unexplored forest, traversed by water streams.

Among the predatory birds nesting in the Cefa Nature Park are: Honey Buzzard Pernis apivorus (one pair), Black Kite Milvus migrans (one pair), Eurasian Sparrowhawk Accipiter nisus (one pair), Common Buzzard Buteo buteo (three to five pairs), Common Kestrel Falco tinnunculus (four to nine), Hobby Falco subbuteo (one to two pairs).

For the Lesser Spotted Eagle Aquila pomarina (one pair) and Peregrine Falcon Falco cherrug (one pair), there were no certain nesting records, but there is an evidence that supports this fact; during the last few years the adult pair was observed throughout the nesting period in the forest area.

Additionally, in Rădvani forest nests also a pair of White-tailed Eagle Haliaetus albicilla.

Of the nocturnal predatory birds nesting here, there are two to three pairs of Longeared Owl Asio otus and one to three pairs of Eurasian Scops Owl Otus scops.

In the Rădvani Forest area, common nesting species are: woodpeckers (Dryocopus martius, Dendrocopos syriacus, D. medius, and Picus canus), wrynecks (Jynx torquilla), pigeons and doves (Stock Dove Columba oenas, Wood Pigeon C. palumbus, and European Turtle Dove Streptopelia turtur), Common Cuckoo Cuculus canorus, Hooded Crow Corvus cornix and many other species of singing birds (Thrush Nightingale Luscinia luscinia, Common Nightingales L. megarhynchos, European Robin Erithacus rubecula, Blackbird Turdus merula, Song Thrush T. philomelos, Mistle Thrush T. viscivorus, Lesser Whitethroat Sylvia curruca, Common Whitethroat S. communis, Barred Warbler S. nisoria, Garden Warbler S. borin, Blackcap S. atricapilla, Wood Warbler Phylloscopus sibilatrix, Common Chiffchaff P. collybita, Willow Warbler P. trochilus, Collared Flycatcher Ficedula albicollis, Spotted Flycatcher Muscicapa striata, Great Tit Parus major, Blue Tit Cyanistes (Parus) caeruleus, Marsh Tit Poecile (Parus) palustris, Goldfinch Carduelis carduelis, Common Starling Sturnus vulgaris, Hawfinch Coccothraustes coccothraustes, Chaffinch Fringilla coelebs, Yellowhammer Emberiza citrinella, Golden Oriole Oriolus oriolus, Greenfinch Carduelis chloris, European Serin Serinus serinus, Woodlark Lullula arborea, Black Redstart Phoenicurus ochruros, Common Redstart P. phoenicurus, and Eurasian Nuthatch Sitta europaea).

Near the Rădvani Forest border area, on the Ateaș locality pasture, nests the European Roller Coracias garrulus (one to three pairs) and also one to two pairs of Eurasian Hoopoe Upupa epops. 


\section{Breeding bird species from fishponds area in Cefa Nature Park}

The vegetation formed on the fishponds constitutes an important habitat for aquatic avifauna. Here the breeding species are: Great Egret Egretta alba (10-15 pairs), Greylag Goose Anser anser, Moorhen Gallinula chloropus, Eurasian Coot Fulica atra, Little Grebe Tachybaptus ruficollis (15-40 pairs) and Black-necked Grebe Podiceps nigricollis (1018 pairs).

On the islets formed by the floating vegetation nests, 240 pairs of Whiskered Tern Chlidonias hybridus, 10 pairs of Black Tern Chlidonias niger and 15-35 pairs of Ferrugineous Duck Aythya nyroca.

Wide reed areas are the optimal habitat for the nesting of 10-12 pairs of Western Marsh Harrier Circus aeruginosus. Moreover, in these areas covered with reed nest the six to nine pairs of Bittern Botaurus stellaris, 40-50 pairs of Little Bittern Ixobrychus minutus, three to seven pairs of Squacco Heron Ardeolla ralloides, eight to nine pairs of Purple Heron Ardea purpurea, and also the Spotted Crake Porzana porzana.

Ducks nest among the pools and in their immediate vicinity: Mallard Anas platyrhynchos (20-30 pairs), Pochard Aythya ferina (75-120 pairs), Garganey Anas querquedula (four to seven pairs), Tufted Duck Aythya fuligula (one to two pairs), Gadwall Anas strepera (10-12 pairs), Red-crested Pochard Neta rufina (one to two pairs).

Some of the birds nesting in reeds and trees on the banks of the canals are as follows: warblers (Great Reed Warbler Acrocephalus arundinaceus, Sedge Warbler A. shoenobaenus, Moustached Warbler A. melanopogon, Marsh Warbler A. palustris, European Reed Warbler A. scirpaceus), Grasshopper Warbler Locustella naevia, River Warbler L. fluviatilis, Savi's Warbler L. luscinioides, Icterine Warbler Hippolais icterina) Reed Bunting Emberiza schoeniclus, Penduline Tit Remiz pendulinus (20-40 pairs) and Bluethroat Luscinia svecica (two to four pairs).

On the banks of the canals which spread all over the Cefa Nature Park are nesting, four to eight pairs of Common Kingfisher Alcedo atthis.

\section{Breeding bird species from meadows, pastures, arable lands and swamps}

These areas are well represented in Cefa Nature Park, the following birds species are nesting here: Northern Lapwing Vanellus vanellus (25-60 pairs), Common Snipe Gallinago gallinago (three to six pairs), White Wagtail Motacilla alba (20-55 pairs), Yellow Wagtail Motacilla flava (70-180 pairs), Black-tailed Godwit Limosa limosa (two to six), Common Redshank Tringa totanus (one to seven pairs).

Over the last years the studied ponds dry up in summer time, thus they are less disturbed and with small height vegetation, or without vegetation. The following bird species nest in this area: Eurasian Curlew Numernius arquata (up to seven pairs), Blackwinged Stilt Himantopus himantopus (up to three pairs), Pied Avocet Recurvirostra avosetta (up to 12 pairs), Common Tern Sterna hirundo (up to 20 pairs), Little Ringed Plover Charadrius dubius (10-16 pairs), and Black-headed Gull Chroicocephalus (Larus) ridibundus (up to 30 pairs).

In the less disturbed grass fields, there were identified nests of Short-eared Owl Asio flammeus.

Agricultural crops from Cefa Nature Park are the nesting place for a series of species of protected birds. Here (towards the Romanian-Hungarian national border) a nest of Montagu's Harrier Circus pygargus was identified, and the presence of another nesting pair of this rare predator is still uncertain. 
Connected to the agricultural crops during their nesting period are also the Common Skylark Alauda arvensis, Tawny Pipit Anthus campestris (four to eight pairs), Corn Bunting Miliaria calandra, and in the bushes bordering the agricultural lands nest the Red-backed Shrike Lanius collurio and Lesser Grey Shrike L. minor (10-12 pairs). In these habitats during nesting period, four pairs of Corncrake Crex crex were identified.

Furthermore, in these habitats the erratic individuals belonging to the Great Bustard Otis tarda were recorded, nesting on the hay fields close to Geszt and Mezőgyán (in the nearby Hungary).

\section{Analysis of threats to the bird fauna in Cefa Nature Park}

There are anthropogenic pressures carried out on the species and habitats today, with different levels of intensity, such as:

\section{Hunting}

Directly affecting all species feeding and nesting on these wetlands. Even though it is done only for the species permitted to be hunted by the national legislation, this activity affects all of the strictly protected species found on these habitats or in the mixed migration and/or feeding groups (Lesser White-Fronted Goose Anser erythropus, Red-Breasted Goose Branta ruficollis, Ferruginous Duck Aythya nyroca, Pygmy Cormorant Phalacrocorax pygmaeus, and Common Sandpiper Actitis hypoleucos etc.).

\section{Fishery management activities}

Inappropriate cutting and/or burning of reeds affects the species nesting in these habitats (warblers, penduline tits, bluethroat, reed buntings, bitterns, etc.). If this burning is done during the migration periods, it can affect the species using the reed as a resting place very seriously during their fall passage (Riparia riparia, Delichon urbica, and Apus apus).

Cutting the natant vegetation formed by Water Chestnut Trapa natans, during nesting (in species such as Aythya nyroca, Chlidonias hybridus, C. niger, and C. leucopterus), has a direct impact on the nesting success of these species that use islets as support for the nests;

Modifying water levels during nesting directly affects the species nesting on the water surface or in its immediate vicinity (Himantopus himantopus, Recurvirostra avosetta, Sterna hirundo, and Chroicocephalus ridibundus), leaving the nests on dry land or flooding the eggs.

\section{Forestry management activities}

Removal of trees in the forest (even dry ones) during the reproduction period directly affects the nesting success of certain species.

Other activities (that may be performed or done in a greater extent) which might affect bird species and their habitats are: intensive sport fishing done in more basins than at present; pasturing intensification or definitive abandonment of it; changing of land use; agricultural activity intensification and especially the use of insecticides might affect the species that are used as food by bird populations; building more vacation houses; the extension of the present land within the built-up area; establishment of industrial development areas in the vicinity; increased access of auto vehicle to the meadows; wind farm development; occurrence of investments in Concentrating Solar Power Technologies; pollution of the aquatic ecosystems because of agriculture, management and intensive fishing; draining and inning of wetlands. 


\section{CONCLUSIONS}

In the habitats from Cefa Nature Park, over half (66\%) of the bird species identified are present with certainty on the territory of Romania (246 species from 373 which are present in Checklist of the Birds of Romania (**).

The area is important both for species reproducing here and also for sedentary and those migratory, providing diverse nesting habitats, distributed on vast areas.

The protected area provides opportunities for refuge, rest and feeding for a variety of migratory species during spring and fall passage and also during winter. Therefore, hundreds of thousands of aquatic and semi-aquatic bird species are present in these seasons in Cefa.

The human pressure is still low in the Cefa Nature Park, which leads the maintenance to be in good to excellent condition of preservation of bird species and their habitats. Even so, the authors' recommendations for the authorities of protected area management are with regard first to the reduction of the anthropogenic pressure intensity represented by hunting and fishpond management (related to maintaining the water level during nesting period, removal of natant vegetation after completion of nesting in the targeted species and mechanic cutting of reeds). 


\section{ACKNOWLEDGEMENTS}

The author is thankful to Cefa Park Adminstration for the support of this study in the field. 


\section{REFERENCES}

1. Bird Directive (79/409/EEC), 1979 - Council Directive on the conservation of wild birds.

2. Bruun B., Delin H. and Svensson L. 1999 - Păsările din România și Europa, Edit. Hamlyn Londra, 1-320. (in Romanian)

3. Curtean-Bănăduc A., Bănăduc D. and Sîrbu I. (eds), 2012 - The Cefa Nature Park, Transylvanian Review of Systematical and Ecological Research, 13, 210.

4. Decision 251/1981 of Bihor County Council. (in Romanian)

5. Law no. 5/2000 regarding the national territory planning, section III, protected areas. (in Romanian)

6. Munteanu D., 2000 - Ornitofauna heleşteelor de la Cefa şi a Pădurii Rădvan, Crisicum, 3, 197 201. (in Romanian)

7. Munteanu D. (ed.), 2004 - Ariile de importanţă avifaunistică din România - documentaţii. Publicația Societății Ornitologice Române, Editura Alma Mater, Cluj-Napoca, 252. (in Romanian)

8. Papp T. and Sándor A. (eds), 2007 - Ariile de Importanţă Avifaunistică din România, Publicația Societății Ornitologice Române și Asociației pentru Protecția Păsărilor și a Naturii “Grupul Milvus”, Tîrgu-Mureş, 252. (in Romanian)

9. Papp T. and Fântânâ C. (eds), 2008 - Ariile de Importanţă Avifaunistică din România, Publicația Societății Ornitologice Române și Asociației pentru Protecția Păsărilor și a Naturii “Grupul Milvus”, Tîrgu-Mureş, 1-319. (in Romanian)

10. Sutherland W. J., Newton I. and Green R. E., 2004 - Bird Ecology and Conservation - A Handbook of Techniques Oxford University Press, Oxford, 1-386.

11. Svensson L., Mullarney K., Zetterstrom D. and Grant P. J., 2010 - Collins Bird Guide, The most complete field guide to the birds of Britain and Europe, HarperCollins, London, UK, 1392.

12. * - Avisabe - The world bird database - last visualization: 25.06.2012. (http://avibase.bsc-eoc.org/avibase.jsp?pg=home\&lang=EN)

13. * - Checklist of the Birds of Romania - last visualization: 25.06.2012. (http://www.milvus.ro/images/PDF/Checklist\%20of\%20the\%20Birds\%20of\%20Romania.pdf) 